\title{
Perda de solo por erosão em decorrência da ação de adubação fosfatada corretiva sobre a estabilidade de agregados
}

\section{Soil erosion as a result of phosphate fertilization on estimated aggregate stability in a typic Acriferric Red Latosol}

\author{
Sérgio Gualberto Martins ${ }^{1 *}$; Marcos Aurélio Carolino de Sá2; Marx Leandro Naves \\ Silva ${ }^{3}$; José Maria de Lima ${ }^{3}$; Anna Hoffmann Oliveira ${ }^{4}$; Júlio César Azevedo \\ Nóbrega ${ }^{5}$ Junior Cesar Avanzi ${ }^{6}$
}

\section{Resumo}

\begin{abstract}
A aplicação de fósforo em solos brasileiros é uma prática frequente e tal operação pode afetar alguns atributos do solo. Assim, com o objetivo de avaliar os efeitos da fosfatagem na estabilidade de agregados e nas perdas de solo por erosão de um Latossolo Vermelho Acriférrico típico de Lavras (MG), foram aplicados, em duas parcelas de $72 \mathrm{~m}^{2}$, o equivalente a $450 \mathrm{~kg} \mathrm{ha}^{-1}$ de $\mathrm{P}_{2} \mathrm{O}_{5}$ a lanço mais $180 \mathrm{~kg} \mathrm{ha}^{-1} \mathrm{de}$ $\mathrm{P}_{2} \mathrm{O}_{5}$ em linhas. A estabilidade de agregados foi determinada por ultra-som para classes de 7,93 a 4,76; 4,76 a 2,00; 2,00 a 1,$00 ; 1,00$ a 0,$50 ; 0,50$ a 0,25 e $<0,25 \mathrm{~mm}$, e por peneiramento úmido para a fração de 7,93 a $4,76 \mathrm{~mm}$. Também foi estimada a perda de solo por erosão com uso do sistema de pinos. A fosfatagem reduziu significativamente a estabilidade de agregados por peneiramento a úmido e por ultrasom para agregados maiores que $1 \mathrm{~mm}$ e aumentou as perdas de solo em 2,85 vezes. Agregados maiores que $2 \mathrm{~mm}$ apresentaram estabilidade 2,83 vezes menor com fosfatagem, o que pareceu influenciar as perdas de solo. A fosfatagem reduziu o ponto de efeito salino nulo e aumentou a densidade de cargas negativas, o que explicou os resultados obtidos.
\end{abstract}

Palavras-chave: Erosão do solo, agregação, ultra-som, fosfatagem

\begin{abstract}
Phosphorus application in Brazilian soils is a common practice and such operation may affect some soil properties. Thus, with the objective of measuring the effect of the phosphate fertilization on aggregate stability and soil loss by erosion of a typic Acriferric Red Latosol from Lavras (MG), soil plots were fertilized with the equivalent to $450 \mathrm{~kg} \mathrm{ha}^{-1}$ of $\mathrm{P}_{2} \mathrm{O}_{5}$, broadcasted, and $180 \mathrm{~kg} \mathrm{ha}^{-1}$ of $\mathrm{P}_{2} \mathrm{O}_{5}$ applied over lines, in two plots of $72 \mathrm{~m}^{2}$. Aggregate stability was measured by sonication at different sizes of aggregates: 7.93 to $4.76 ; 4.76$ to $2.00 ; 2.00$ to $1.00 ; 1.00$ to $0.50 ; 0.50$ to 0.25 and $<0.25 \mathrm{~mm}$. Wet sieving was also performed to estimate aggregate stability at the fraction of 7.93 to $4.76 \mathrm{~mm}$. The amount of erosion was measured using a grid of pins on soil surface. Phosphate fertilization significantly reduced aggregate stability by wet sieving and ultrasonic for larger aggregates than $1 \mathrm{~mm}$, and increased the erosion in 2.85 times. Aggregates larger than $2 \mathrm{~mm}$ showed stability 2.83 times lower with phosphate, which could influence soil loss. The phosphate fertilization reduced the zero point of salt effect, and increased the negative charges density,
\end{abstract}

1 Dr. em Ciência do Solo, Prof. do Centro Universitário de Lavras, UNILAVRAS. E-mail: sergio.gualberto@unilavras.edu.br

2 Dr. em Ciência do Solo, Pesquisador da Embrapa Cerrados. E-mail: carolino@cpac.embrapa.br

3 Dr. em Ciência do Solo, Profs. do Departamento de Ciência do Solo, Universidade Federal de Lavras, UFLA. E-mail: marx@ dcs.ufla.br; jmlima@dcs.ufla.br

${ }^{4}$ M. Sc. em Ciência do Solo, Doutoranda em Ciência do Solo, UFLA. E-mail: anna.ufla@gmail.com

5 Dr. em Ciência do Solo, Prof. do Departamento de Ciência do Solo, UFPI. E-mail: jnobrega@ufpi.br

6 Dr. em Ciência do Solo, Pesquisador da Embrapa Pesca, Aquicultura e Sistemas Agrícolas. E-mail: junior.avanzi@embrapa.br

* Autor para correspondência 
which helps to explain these results.

Key words: Soil erosion, aggregation, ultrasound, phosphate fertilization

\section{Introdução}

A agregação do solo tem efeito direto em propriedades de interesse agronômico, como a distribuição do tamanho de poros, condutividade hidráulica, susceptibilidade à compactação e, consequentemente, à erosão do solo. Na busca por sistemas de manejo de solo agrícola sustentáveis, uma das prioridades é promover a sua conservação, que é alcançada pela manutenção e, às vezes, formação de agregados estáveis (CHAPPELL; TERNAN; BIDIN, 1999), entre outros aspectos. O manejo conservacionista favorece a formação e a estabilidade de agregados do solo (CASTRO FILHO et al., 2002; PINHEIRO; FEREIRA; ANJOS, 2004; ANNABI et al., 2007; KASPER et al., 2009) e, esta propriedade, aliada à densidade e porosidade do solo, determina, em parte, a qualidade da estrutura e regula a velocidade e o grau de sua degradação (BERTOL et al., 2006).

Os agregados ocorrem naturalmente, com a união de partículas do solo em microagregados que, juntos, formam os macroagregados (TISDALL; OADES, 1982). Os agregados são de grande importância para a conservação do solo por conferirem maior resistência ao processo erosivo e proteção à matéria orgânica (FERREIRA; TAVARES FILHO; FERREIRA, 2010). Encontram-se, na literatura, referências segundo as quais a aplicação de adubos e corretivos ao solo, além das ações mecânicas, pode contribuir tanto para a formação quanto para a destruição dos agregados (LIMA; ANDERSON; CURI, 2000). Isto decorre do fato de que certos compostos químicos, quando adicionados ao solo, contribuem para alterar a densidade de cargas da superfície dos colóides. Este fato é mais relevante em solos mais intemperizados, os quais têm suas cargas originadas pela protonação e deprotonação, com influência da adsorção desses íons principalmente na superfície de óxidos e hidróxidos de $\mathrm{Fe}$ e Al
(RANNO et al., 2007). Esse efeito pode alterar atributos físico-químicos relacionados à dispersão de partículas, além de aumentar a lixiviação e a retenção de nutrientes, encrostamento superficial e agregação do solo, consequentemente, reduzindo a resistência do solo à erosão.

No caso específico do fosfato, sua fixação ou retenção pela superfície de minerais e/ou precipitação com outros elementos presentes na solução do solo, especialmente em latossolos mais oxídicos, tem levadoà necessidade de pesadas adubações (RANNO et al., 2007; SANTOS; GATIBONI; KAMINSKI, 2008). Neste sentido, dependendo do tipo de carga predominante na superfície das partículas do solo, a adubação fosfatada pode contribuir tanto para aumentar quanto para diminuir a dispersão de tais partículas, alterando a estabilidade dos agregados. As alterações decorrentes da aplicação de certos compostos químicos ao solo, a exemplo do fosfato, podem depender não apenas dos produtos adicionados e de suas quantidades, mas também da classe de solo com suas características físicas, químicas e mineralógicas.

O presente trabalho teve como objetivo, verificar o efeito da adubação fosfatada corretiva sobre o índice de estabilidade dos agregados, determinado através de ultra-som e peneiramento úmido, e sua relação com as perdas de solo por erosão hídrica em um Latossolo Vermelho Acriférrico típico da região de Lavras (MG).

\section{Material e Métodos}

Em um Latossolo Vermelho Acriférrico típico fase Cerrado do município de Lavras (MG) com 8\% de declive, foram instaladas 4 parcelas de $72 \mathrm{~m}^{2}(6$ $\mathrm{x} 12 \mathrm{~m}$ ), cujo maior comprimento foi disposto no sentido de maior declividade do terreno, mantidas capinadas nos 6 meses anteriores a instalação do 
experimento. Nas duas parcelas centrais (tratamento 1), foram aplicados o equivalente a $450 \mathrm{~kg} \mathrm{ha}^{-1}$ de $\mathrm{P}_{2} \mathrm{O}_{5}$ a lanço, incorporado na profundidade de 0 a $5 \mathrm{~cm}$, mais $180 \mathrm{~kg} \mathrm{ha}^{-1}$ de $\mathrm{P}_{2} \mathrm{O}_{5}$ em linhas, à superfície do terreno, simulando uma fosfatagem pesada na implantação de uma cultura, com espaçamento de $40 \mathrm{~cm}$. Em ambos os casos, foi usado o fosfato de potássio como fonte de fósforo. O processo de revolvimento da camada de 0 a $5 \mathrm{~cm}$ para incorporação do adubo fosfatado foi também realizado no tratamento sem fosfatagem (tratamento 2), visando igualar as condições. Portanto, para cada tratamento foram feitas duas repetições no campo.

As perdas de solo por erosão sob chuva natural, foram estimadas pela diminuição de nível na superfície do solo (SANTOS et al., 1998), com a medição por meio de pinos metálicos de $20 \mathrm{~cm}$ de comprimento, cravados no solo até $15 \mathrm{~cm}$ de profundidade, em 60 pontos de cada parcela. Os pinos foram colocados 15 dias após a incorporação do adubo, admitindo-se ser este um período suficiente para acamamento e estabilização da superfície do solo. As medições foram feitas diretamente nos pinos a cada 10 dias com aproximação de $1 \mathrm{~mm}$, perfazendo um total de 6 medições no período de 50 dias entre os meses de janeiro e fevereiro de 1998. Neste período ocorreram $308 \mathrm{~mm}$ de precipitação. Para estimar as perdas de solo usou-se a seguinte expressão:

$$
P=H x A x D s
$$

Em que, é a perda de solo $\left(\mathrm{t} \mathrm{ha}^{-1}\right)$; é a média das alterações de nível da superfície do solo medida nos pinos $(\mathrm{m})$; é a área considerada, equivalente a um hectare $\left(10.000 \mathrm{~m}^{2}\right)$; e é a densidade do solo $\left(\mathrm{t} \mathrm{m}^{-3}\right)$.

Decorrido um período de 150 dias após a adubação, foram coletadas amostras de agregados de solo em cada parcela a uma profundidade de 0-5 cm que, posteriormente, foram secas à sombra e cuidadosamente peneiradas, separando-se agregados nas seguintes classes de diâmetro: 7,93 a 4,$76 ; 4,76$ a 2,$00 ; 2,00$ a 1,$00 ; 1,00$ a 0,$50 ; 0,50$ a 0,25 e $<0,25 \mathrm{~mm}$.
$\mathrm{Na}$ classe de 7,93 a 4,76 mm, foi avaliada a distribuição dos agregados estáveis ao peneiramento úmido utilizando-se amostras previamente umedecidas, calculando-se o diâmetro médio geométrico (DMG) e o diâmetro médio ponderado (DMP), segundo Kemper e Chepil (1965). Para cada classe de diâmetro, foi também determinado o pH em água, carbono orgânico e complexo sortivo, apenas para caracterização, em subamostras passadas em peneira de $2 \mathrm{~mm}$, quando necessário.

A densidade do solo (Ds) foi obtida utilizandose o método do torrão parafinado, segundo Black e Hartge (1986). Amostras do solo foram também destorroadas e passadas em peneira de $2 \mathrm{~mm}$ (TFSA), para determinação do ponto de efeito salino nulo (PESN), segundo Zelasny, He e Vanwormhoudt (1996), com utilização do método gráfico, segundo Silva et al. (1996). Também foram determinados, na mesma fração, a granulometria (DAY, 1965), o índice de floculação de partículas e o pH em água e em KCl, segundo EMBRAPA (1997). Todas as determinações laboratoriais foram feitas com três repetições.

Para as medições ao ultra-som foi necessária a calibração prévia da potência emitida pelo aparelho, baseado em técnicas calorimétricas. Para cada fração de agregados, foi determinada a curva de dispersão por ultra-som, comparando-se a estabilidade de agregados nas condições sem e com fosfatagem, conforme metodologia proposta por Sá et al. (1999). Este procedimento consistiu em tomar amostras (3 repetições) correspondente a $10 \mathrm{~g}$ de agregados (peso seco em estufa, sob temperatura de $105^{\circ} \mathrm{C}$ ) e submetê-las a um pré-umedecimento por capilaridade durante duas horas, em bandeja com areia úmida coberta por uma folha de papel de filtro. Cada amostra foi sonificada em béquer com $400 \mathrm{~mL}$ de água destilada (dando uma relação solo:água destilada de 1:40), durante 5, 15, 30, $60,300,900,1800$ e $2700 \mathrm{~s}$, o que corresponde às energias específicas aplicadas de 1,32; 3,97; 7,94; 15,$88 ; 79,42 ; 238,27 ; 476,53$ e $714,80 \mathrm{~J} \mathrm{~mL}^{-1}$. 
A ponta da haste do sonificador foi inserida na suspensão a uma profundidade de $85 \mathrm{~mm}$ e, para reduzir o efeito da alta temperatura na amostra, esta foi colocada em banho de gelo durante a sonificação, ficando a temperatura da suspensão abaixo de 40 ${ }^{\circ} \mathrm{C}$, conforme sugerido por Gregorich, Kachanoski e Voroney (1989). Após cada sonificação, as amostras foram cuidadosamente passadas em peneira de $0,053 \mathrm{~mm}$, quantificando a fração dispersa (silte + argila) por diferença (massa inicial de agregados menos a fração retida na peneira, ambos massa seca em estufa sob temperatura de $105^{\circ} \mathrm{C}$ ). Foi, então, calculado o índice de dispersão (fração < 0,053 $\mathrm{mm} /$ massa inicial de agregados secos), para cada tempo de sonificação. Os resultados foram plotados em curvas de dispersão, sendo na abcissa a energia específica aplicada e na ordenada o índice de dispersão. As curvas foram linearizadas e ajustadas matematicamente, conforme Sá et al. (1999). A linearização foi feita plotando-se no eixo das abcissas o nível de energia específica aplicado à suspensão, em $\mathrm{J} \mathrm{mL}^{-1} \mathrm{e}$, no eixo das ordenadas, esse mesmo nível de energia dividido pelo índice de dispersão, ajustando-se uma equação do tipo $y=a+b x$ através de regressão. O termo $b / a$, cuja unidade é $\mathrm{mL} \mathrm{J}^{-1}$, foi usado como um índice para expressar a susceptibilidade do solo à desagregação, uma vez que representa o comportamento da curva que considera todos os níveis de energia aplicados. Assim, o modelo ajustado foi uma equação do tipo $y=[x /(a+b x)]$, onde y é o índice de dispersão e é a energia aplicada à amostra, em $\mathrm{J} \mathrm{mL}^{-1} ; a$ e $b$ são os coeficientes da equação. Na análise estatística, os coeficientes $a$ e $b$ das equações foram comparados para as duas condições, dentro de cada fração de agregados, com uso do teste de F considerando como diferentes os índices $b / a$ quando o teste mostrou diferenças entre os coeficientes lineares e angulares, para as duas condições. Para o peneiramento úmido, os resultados foram comparados utilizando-se o teste de Tuckey a 5\%.

\section{Resultados e Discussão}

Na Figura 1 está apresentado o efeito da adubação fosfatada sobre alguns parâmetros físicos do solo estudado. Verifica-se que o índice de floculação de partículas não foi alterado pela fosfatagem, ao contrário do DMP e DMG, com menores valores nas parcelas com fosfato, mostrando que a adubação fosfatada na dose utilizada diminuiu a estabilidade dos agregados, conforme também verificado por outros pesquisadores (LIMA; ANDERSON; CURI, 2000; ALBUQUERQUE et al., 2003).

Tabela 1. Granulometria, índice de floculação, densidade do solo (Ds), diâmetro médio geométrico (DMG), diâmetro médio ponderado (DMP) em Latossolo Vermelho Acriférrico típico sem e com fosfatagem.

\begin{tabular}{|c|c|c|c|c|c|c|c|}
\hline \multirow[t]{2}{*}{ Tratamento } & \multicolumn{3}{|c|}{ Granulometria } & \multirow{2}{*}{$\begin{array}{c}\text { Índice de } \\
\text { Floculação }\end{array}$} & \multirow[t]{2}{*}{ DS } & \multirow[t]{2}{*}{${ }^{1} \mathrm{DMG}$} & \multirow[t]{2}{*}{${ }^{1} \mathbf{D M P}$} \\
\hline & Argila & Silte & $\overline{\text { Areia }}$ & & & & \\
\hline & \multicolumn{3}{|c|}{--------g kg-1------------ } & ----\% \%---- & $\mathrm{g} \mathrm{cm}^{-3}$ & \multicolumn{2}{|c|}{--------mm-------- } \\
\hline Sem fosfatagem & 390 & 310 & 300 & $84 \mathrm{a}$ & 1,02 & $4,32 \mathrm{a}$ & $4,60 \mathrm{a}$ \\
\hline Com fosfatagem & 420 & 320 & 260 & $86 \mathrm{a}$ & 1,02 & $3,57 \mathrm{~b}$ & $4,33 \mathrm{~b}$ \\
\hline$\overline{\mathrm{CV} \%}$ & - & - & - & 2,35 & - & 12,31 & 4,93 \\
\hline DMS & - & - & - & 5,23 & - & 0,15 & 0,21 \\
\hline
\end{tabular}

${ }^{1}$ Agregados entre 7,93-4,76 mm - peneiramento úmido. Médias seguidas da mesma letra na coluna não diferem entre si pelo teste de Tukey $5 \%$. 
Observa-se na Tabela 2 uma semelhança nos teores de carbono orgânico entre as classes estudadas. Os teores de $\mathrm{K}$ foram maiores nas frações de agregados da parcela que recebeu a fosfatagem, uma vez que a fonte de fósforo utilizada continha potássio. Entretanto, provavelmente este elemento não tenha afetado a estabilidade de agregados, pois mesmo sendo um cátion monovalente, este não é tão facilmente hidratável como o sódio ou o lítio, não sendo, portanto, um dispersante efetivo (HILLEL, 1982).

Tabela 2. Carbono orgânico, $\mathrm{pH}$ e complexo sortivo para cada classe de agregado do Latossolo Vermelho Acriférrico típico sem (SP) e com adubação fosfatada (CP).

\begin{tabular}{|c|c|c|c|c|c|c|c|c|c|c|c|c|}
\hline \multirow[t]{3}{*}{ Atributos } & \multicolumn{12}{|c|}{ Frações de agregados $(\mathrm{mm})$} \\
\hline & \multicolumn{2}{|c|}{$7,93-4,76$} & \multicolumn{2}{|c|}{$4,76-2,00$} & \multicolumn{2}{|c|}{$2,00-1,00$} & \multicolumn{2}{|c|}{$\mathbf{1 , 0 0}-\mathbf{0 , 5 0}$} & \multicolumn{2}{|c|}{$0,50-0,25$} & \multicolumn{2}{|c|}{$<0,25$} \\
\hline & SP & $\mathbf{C P}$ & $\mathbf{S P}$ & $\mathbf{C P}$ & SP & $\mathbf{C P}$ & SP & $\mathbf{C P}$ & SP & $\mathbf{C P}$ & SP & $\mathbf{C P}$ \\
\hline $\mathrm{K}\left(\mathrm{mg} \mathrm{dm}^{-3}\right)$ & 25,6 & 152,0 & 22,6 & 157,0 & 16,7 & 132,0 & 17,0 & 132,0 & 20,0 & 142,0 & 23,0 & 151,0 \\
\hline $\mathrm{P}\left(\mathrm{mg} \mathrm{dm} \mathrm{dm}^{-3}\right)$ & 1,0 & 3,3 & 1,0 & 3,0 & 1,0 & 3,3 & 1,0 & 4,7 & 1,0 & 4,0 & 1,0 & 5,3 \\
\hline $\mathrm{C}\left(\mathrm{g} \mathrm{kg}^{-1}\right)$ & 18 & 19 & 21 & 21 & 19 & 19 & 14 & 15 & 17 & 17 & 21 & 21 \\
\hline $\mathrm{PH}$ & 5,7 & 5,9 & 5,8 & 5,9 & 5,8 & 5,9 & 5,8 & 6,0 & 5,8 & 5,9 & 5,8 & 5,9 \\
\hline $\mathrm{H}+\mathrm{Al}\left(\mathrm{mmol}_{\mathrm{c}} \mathrm{dm}^{-3}\right)$ & 5,0 & 4,7 & 4,0 & 4,7 & 3,0 & 3,7 & 3,9 & 4,0 & 4,0 & 4,7 & 5,2 & 4,8 \\
\hline $\mathrm{S}\left(\mathrm{mmol}_{\mathrm{c}} \mathrm{dm}^{-3}\right)$ & 4,3 & 3,4 & 4,2 & 3,4 & 3,4 & 2,8 & 3,5 & 3,3 & 3,6 & 2,9 & 4,0 & 3,4 \\
\hline $\mathrm{t}\left(\mathrm{mmol}_{\mathrm{c}} \mathrm{dm}^{-3}\right)$ & 4,3 & 3,4 & 4,2 & 3,4 & 3,4 & 2,8 & 3,5 & 3,3 & 3,6 & 2,9 & 4,0 & 3,4 \\
\hline $\mathrm{T}\left(\mathrm{mmol}_{\mathrm{c}} \mathrm{dm}^{-3}\right)$ & 6,3 & 8,1 & 8,2 & 8,0 & 6,4 & 6,6 & 7,4 & 7,3 & 7,6 & 7,6 & 9,2 & 8,2 \\
\hline$V(\%)$ & 46,3 & 42,4 & 51,0 & 42,0 & 52,9 & 43,3 & 47,5 & 45,1 & 47,3 & 38,8 & 43,5 & 41,5 \\
\hline
\end{tabular}

Os valores de $\mathrm{pH}$ foram mais elevados nas parcelas que receberam fosfatagem, embora esta diferença tenha sido de apenas 0,1 ou 0,2 unidade (Tabela 2). Isso se deve ao deslocamento de grupos $\mathrm{OH}$ da superfície das partículas pelo ânion fosfato, no processo chamado troca de ligantes.

Assim como observado por Sá, Lima e Mello (2002), houve uma tendência das curvas à formação de um patamar a partir de $90 \mathrm{~J} \mathrm{~mL}^{-1}$ (Figura 1). Segundo estes autores, a melhor faixa de energia ultra-sônica para se avaliar a estabilidade de agregados em Latossolo Vermelho acriférrico está entre 30 e $90 \mathrm{~J} \mathrm{~mL}^{-1}$. Visualmente, diferenças na estabilidade dos agregados e entre as curvas ajustadas para as classes 7,93 - 4,76 e 4,76 - 2,00 mm, são observadas apenas em níveis de energia inferiores a $400 \mathrm{~J} \mathrm{~mL}^{-1}$, confirmando as informações de Sá, Lima e Mello (2002) as quais relatam que níveis mais altos de energia ultra sônica não são adequados para determinar diferenças na estabilidade de agregados. Para as classes de menor diâmetro, as curvas praticamente se sobrepõem, evidenciando maior efeito do fosfato nos macroagregados (Figura 1).

$\mathrm{Na}$ Tabela 3 estão apresentadas as equações linearizadas $(y=a+b x)$, seus respectivos coeficientes de determinação e a comparação entre seus coeficientes. Os modelos ajustados para as curvas de dispersão foram $\mathrm{y}=\mathrm{x} /(\mathrm{a}+\mathrm{bx})$, conforme sugerido por Sá et al. (1999). Em todos os casos, os modelos ajustados apresentaram valores de $r^{2}$ altamente significativos. Diferenças entre 
coeficientes lineares e angulares para os tratamentos sem e com fosfatagem, foram constatadas somente nas classes de agregados maiores que $2 \mathrm{~mm}$. Isto se deve ao efeito da adsorção do íon fosfato sobre o balanço de cargas das partículas e, portanto, na estabilidade dos agregados, concordando com Wann e Uehara (1978) e Lima, Anderson e Curi (2000).

Figura 1. Curvas de estabilidade de agregados por ultra-som, para cada classe de agregados de um Latossolo Vermelho Acriférrico típico sem e com adubação fosfatada.

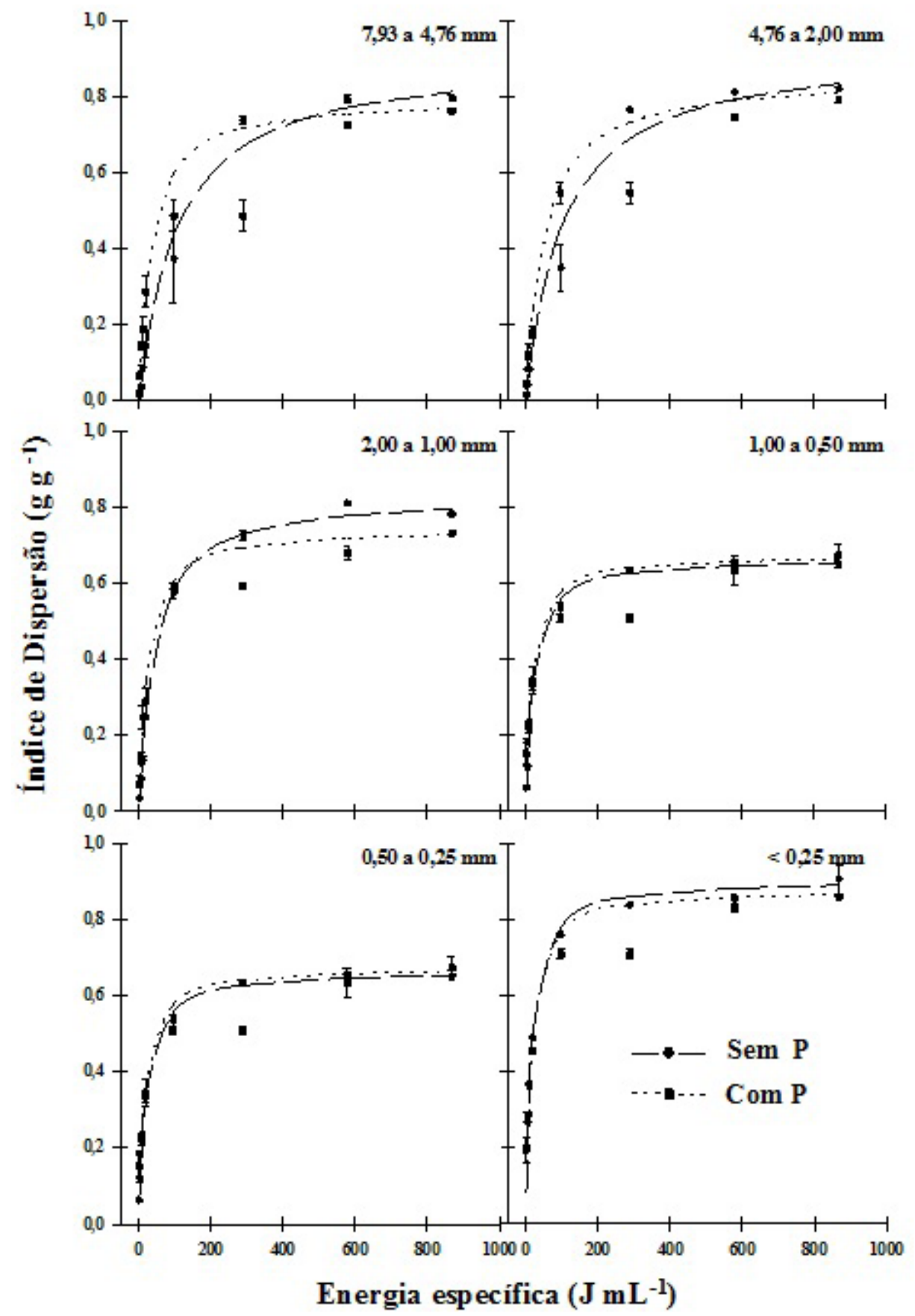


Na classe entre 1 e $2 \mathrm{~mm}$ também foi observada diferença significativa, mas apenas entre os coeficientes angulares, mostrando diferença entre a inclinação das curvas linearizadas. Para as classes de agregados abaixo de $1 \mathrm{~mm}$, não foram observadas diferenças entre os coeficientes das equações, mostrando que o fosfato influencia significativamente a estabilidade dos agregados, mas apenas nas classes maiores que $1 \mathrm{~mm}$. Assim como os microagregados são dificilmente afetados pelo manejo, conforme sugerido por Tisdall e Oades (1982), algumas classes de macroagregados $(1,00-$ $0,50 ; 0,50-0,25)$, também não foram afetadas pela fosfatagem.

Tabela 3. Modelos lineares ajustados, valores de r2 e comparação entre os coeficientes das equações para cada classe de diâmetro de agregado de um Latossolo Vermelho Acriférrico típico sem (SP) e com adubação fosfatada (CP).

\begin{tabular}{llccc}
\hline $\begin{array}{l}\text { Frações } \\
(\mathbf{m m})\end{array}$ & \multicolumn{1}{c}{$\mathbf{S P}$} & $\mathbf{r}^{2}$ & \multicolumn{1}{c}{$\mathbf{C P}$} & $\mathbf{r}^{2}$ \\
\hline $7,93-4,76$ & $\mathrm{y}=1,0799 \mathrm{x}+128,2588$ & $0,968 * *$ & $\mathrm{y}=1,2517 \mathrm{x}+41,9602$ & $0,997 * *$ \\
$4,76-2,00$ & $\mathrm{y}=1,0672 \mathrm{x}+112,4725$ & $0,983 * *$ & $\mathrm{y}=1,1658 \mathrm{x}+58,0115$ & $0,998 * *$ \\
$2,00-1,00$ & $\mathrm{y}=1,1966 \mathrm{x}+50,1578$ & $0,998 * *$ & $\mathrm{y}=1,3383 \mathrm{x}+30,4098$ & $0,997 * *$ \\
$1,00-0,50$ & $\mathrm{y}=1,4953 \mathrm{x}+28,7972$ & $0,999 * *$ & $\mathrm{y}=1,4762 \mathrm{x}+25,3446$ & $0,997 * *$ \\
$0,50-0,25$ & $\mathrm{y}=1,2642 \mathrm{x}+43,8136$ & $0,995 * *$ & $\mathrm{y}=1,2592 \mathrm{x}+45,6926$ & $0,994 * *$ \\
$<0,25$ & $\mathrm{y}=1,1047 \mathrm{x}+17,1448$ & $0,996 * *$ & $\mathrm{y}=1,1340 \mathrm{x}+16,4597$ & $0,999 * *$ \\
\hline
\end{tabular}

\section{Comparação entre coeficientes}

\begin{tabular}{lllllll} 
& \multicolumn{9}{c}{ Linear } & \multicolumn{5}{c}{ Angular } \\
\cline { 2 - 7 } & SP & CP & Sign. & SP & CP & Sign. \\
\hline $7,93-4,76$ & 128,2588 & 41,9602 & $* *$ & 1,0799 & 1,2517 & $* *$ \\
$4,76-2,00$ & 112,4725 & 58,0115 & $* *$ & 1,0672 & 1,1658 & $* *$ \\
$2,00-1,00$ & 50,1578 & 30,4098 & ns & 1,1966 & 1,3383 & $* *$ \\
$1,00-0,50$ & 28,7972 & 25,3446 & ns & 1,4953 & 1,4762 & ns \\
$0,50-0,25$ & 43,8136 & 45,6926 & ns & 1,2642 & 1,2592 & ns \\
$<0,25$ & 17,1448 & 16,4597 & ns & 1,1047 & 1,1340 & ns \\
\hline
\end{tabular}

** Significativo pelo teste de F de Snedecor e Cochran (1989) ao nível de 1\%; ns = não significativo.

Conforme verificado por Adesodun, Adeyemi e Oyegoke (2007), a distribuição do fósforo avaliado na análise de estabilidade de agregados em água mostra um enriquecimento preferencial de fósforo na classe de macroagregados $(>0,25 \mathrm{~mm})$ para solos não cultivados e na classe de microagregados para solos cultivados. Considerando que o cultivo reduz as classes de macroagregados para diâmetros menores e que pequenos agregados são preferencialmente removidos pela erosão hídrica, é clara a necessidade de práticas sustentáveis de manejo de solo que possam minimizar a perda de nutrientes.

Para os índices de desagregação por ultra-som (b/a) (SÁ et al., 1999), apresentados no (Tabela 4), observa-se que, em geral, os valores foram mais 
elevados onde realizou-se fosfatagem (1,77 vezes). O índice foi 2,50 vezes maior para as classes onde a adubação fosfatada alterou significativamente a inclinação (7,93 - 4,76; 4,76 - 2,00 e 2,00 - 1,00) e 2,83 vezes maior nas classes de tamanho acima de $2 \mathrm{~mm}$, onde foram observadas diferenças entre todos os coeficientes das equações ajustadas. Essa maior desagregação de partículas no tratamento com adubação fosfatada refletiu diretamente no aumento das perdas de solo por erosão (Tabela 4), que foram equivalentes a 41,3 $\mathrm{t} \mathrm{ha}^{-1}$, ou seja, 2,85 vezes maior que o tratamento sem fosfatagem, que teve perda equivalente a $14,5 \mathrm{t} \mathrm{ha}^{-1}$. Considerandose que a relação índice de desagregação $(b / a)$ para agregados acima de $2 \mathrm{~mm}(2,83)$ está bem próxima da relação de perdas de solo $(2,85)$, pode-se inferir que a estabilidade dos agregados maiores que $2 \mathrm{~mm}$ tem maior influência no processo erosivo.

Tabela 4. Índices de desagregação por ultra-som $(b / a)$, porcentagem de agregados estáveis ao peneiramento úmido para a classe 7,93 - 4,76 mm e valores de perdas de solo em um Latossolo Vermelho Acriférrico típico sem (SP) e com adubação fosfatada (CP).

\begin{tabular}{|c|c|c|c|c|c|}
\hline \multirow{3}{*}{$\begin{array}{l}\text { Classes } \\
\mathrm{mm} \\
\end{array}$} & \multicolumn{2}{|c|}{ Índice $b / a$} & \multirow{3}{*}{$\begin{array}{l}\text { Relação do índice } \\
\text { CP/SP }\end{array}$} & \multicolumn{2}{|c|}{ Peneiramento Úmido } \\
\hline & SP & $\mathbf{C P}$ & & SP & $\mathbf{C P}$ \\
\hline & \multicolumn{2}{|c|}{ - $\left(\mathrm{mL} \mathrm{J}^{-1}\right)-\overline{-}$} & & \multicolumn{2}{|c|}{ 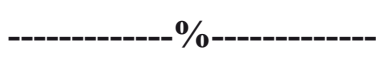 } \\
\hline $7,93-4,76$ & 0,0084 & 0,0298 & 3,54 & - & - \\
\hline $4,76-2,00$ & 0,0095 & 0,0201 & 2,11 & $36 \dagger$ & $28 \dagger$ \\
\hline $2,00-1,00$ & 0,0239 & 0,0440 & 1,84 & 13 & 16 \\
\hline $1,00-0,50$ & 0,0519 & 0,0582 & 1,12 & 20 & 25 \\
\hline $0,50-0,25$ & 0,0289 & 0,0276 & 0,95 & 16 & 18 \\
\hline$<0,25$ & 0,0644 & 0,0689 & 1,06 & 15 & 13 \\
\hline${ }^{1}$ Média geral & & & 1,77 & - & - \\
\hline${ }^{2}$ Média > 1 mm & & & 2,50 & - & - \\
\hline${ }^{3}$ Média > $2 \mathrm{~mm}$ & & & 2,83 & - & - \\
\hline \multicolumn{3}{|c|}{ Perdas de solo por erosão $\left(\mathrm{t} \mathrm{ha}^{-1}\right)$} & \multicolumn{2}{|c|}{ Relação CP/SP } & \\
\hline $\mathrm{CP}$ & SP & & & & \\
\hline 41,3 & 14,5 & & & & \\
\hline
\end{tabular}

Índices de desagregação seguidos de mesma letra, na mesma linha, não diferem entre si pelo teste de Tukey $5 \%$.

$\dagger$ Agregados entre 7,93-2,00 mm.

${ }^{1}$ Média dos índices b/a para todas as classes de agregados.

${ }^{2}$ Média dos índices b/a apenas para as classes de agregados onde foi observada diferença significativa entre os coeficientes lineares das equações (diâmetro acima de $1 \mathrm{~mm}$ ).

${ }^{3}$ Média dos índices b/a apenas para as classes de agregados onde foi observada diferença significativa entre todos os coeficientes das equações (diâmetro acima de $2 \mathrm{~mm}$ ).

$\mathrm{Na}$ Tabela 5 podem ser observados os valores do ponto de efeito salino nulo (PESN), pH em água e $\mathrm{KCl}$ e valores de $\Delta \mathrm{pH}$. Verifica-se que a adubação fosfatada reduziu o valor do PESN em 0,4 unidades de $\mathrm{pH}$. Essa diferença, embora pequena, mostra um ligeiro incremento na densidade de cargas negativas, confirmado pelos valores de $\Delta \mathrm{pH}$ que, aparentemente, explicam a redução da estabilidade 
dos agregados, tanto ao peneiramento úmido quanto ao ultra-som, e o consequente aumento das perdas de solo por erosão. A redução na estabilidade dos agregados ao peneiramento úmido pode ser atribuída à adsorção de fosfato, que altera o balanço de cargas do solo (LIMA; ANDERSON; CURI, 2000; ALBUQUERQUE et al., 2003), promovendo dispersão de partículas, o que pode ser explicado pelas alterações no ponto de efeito salino nulo e $\Delta \mathrm{pH}$ (Tabela 5), uma vez que o teor de carbono orgânico (Tabela 2), também responsável pela formação e estabilidade de agregados do solo (ANNABI et al., 2007; ABID; LAL, 2008; KASPER et al., 2009), foi semelhante entre as classes estudadas.

Tabela 5. Ponto de efeito salino nulo (PESN), $\mathrm{pH}$ em água, $\mathrm{KCl}$ e $\Delta \mathrm{pH}$ determinados na terra fina seca ao ar de um Latossolo Vermelho Acriférrico típico sem e com adubação fosfatada.

\begin{tabular}{lcccc}
\hline Tratamento & PESN & \multicolumn{2}{c}{$\mathbf{p H}$} & \multicolumn{2}{c}{$\Delta \mathbf{p H}$} \\
& & $\mathbf{H}_{2} \mathbf{O}$ & $\mathbf{K C l} \mathbf{1} \mathbf{~ m o l ~ \mathbf { L } ^ { - 1 }}$ & \\
\hline Sem fosfatagem & 5,0 & 5,77 & 4,91 & $-0,86$ \\
Com fosfatagem & 4,6 & 5,96 & 4,94 & $-1,02$ \\
\hline
\end{tabular}

Os aspectos aqui discutidos evidenciaram que a adubação fosfatada deve ser levada em consideração, quando se discutem fatores que contribuem para a redução da estabilidade de agregados e aumento das perdas de solo por erosão. Tal fato deve ser considerado principalmente na realização de fosfatagem em sistemas de cultivo em que, todos os anos, o solo é mantido exposto do final do preparo até a fase em que a cultura inicie a cobertura do terreno, onde práticas conservacionistas eficientes serão necessárias.

\section{Conclusões}

1. A erosão hídrica do solo aumenta com a diminuição da estabilidade dos agregados maiores que $2 \mathrm{~mm}$.

2. A adubação fosfatada corretiva aumenta as cargas negativas superficiais das argilas, reduz a estabilidade dos agregados maiores que $1 \mathrm{~mm}$ e aumenta as perdas de solo por erosão hídrica.

\section{Referências}

ABID, M.; LAL, R. Tillage and drainage impact on soil quality. I. Aggregate stability, carbon and nitrogen pools. Soil \& Tillage Research, Amsterdam, v. 100, n. 1/2, p. 89-98, jul./aug. 2008.

ADESODUN, J. K.; ADEYEMI, E. F.; OYEGOKE, C. $O$. Distribution of nutrient elements within water-stable aggregates of two tropical agro-ecological soils under different land uses. Soil \& Tillage Research, Amsterdam, v. 92, n. 1/2, p. 190-197, jan. 2007.

ALBUQUERQUE, J. A.; BAYER, C.; ERNANI, P. R.; MAFRA, A. L.; FONTANA, E. C. Aplicação de calcário e fósforo e estabilidade da estrutura de um solo ácido. Revista Brasileira de Ciência do Solo, Viçosa, v. 27, n. 5, p. 799-806, out. 2003.

ANNABI, M.; HOUOT, S.; FRANCOU, C.; POITRENAUD, M.; BISSONNAIS, Y. L. Soil aggregate stability improvement with urban compost of different maturities. Soil Science Society of America Journal, Madison, v. 71, n. 2, p. 413-423, mar./apr. 2007.

BERTOL, I.; AMARAL, A. J.; VÁSQUEZ, E. V.; GONZÁLEZ, A. P.; BARBOSA, F. T.; BRIGNONI, L. F. Relações da rugosidade superficial do solo com o volume de chuva e com a estabilidade de agregados em água. Revista Brasileira de Ciência do Solo, Viçosa, v. 90, n. 3, p. 543-553, jun. 2006.

BLACK, G. R.; HARTGE, K. H. Bulk density. In: KLUTE, A. (Ed.). Methods of soil analysis: physical and mineralogical methods. 2. ed. Madison: American 
Society of Agronomy, 1986. p. 363-375.

CASTRO FILHO, C.; LOURENÇO, A.; GUIMARÃES, M. F.; FONSECA, I. C. B. Aggregate stability under different soil managment systems in a red latosol in the state of Parana, Brazil. Soil \& Tillage Research, Amsterdam, v. 65, n. 1, p. 45-51, may 2002.

CHAPPELL, N. A.; TERNAN, J. L.; BIDIN, K. Correlation of physicochemical properties and suberosional landforms with aggregate stability variations in a tropical Ultisol disturbed by forestry operations. Soil \& Tillage Research, Amsterdam, v. 50, n. 1, p. 55-71, 1999.

DAY, P. R. Particle fractionation and particle-size analysis. In: BLACK, C. A. (Ed.). Methods of soil analysis. Part 1. Madison: American Society of Agronomy Inc., Wisconsin, USA, 1965. p. 545-566.

EMPRESA BRASILEIRA DE PESQUISA AGROPECUÁRIA - EMBRAPA. Centro Nacional de Pesquisa de Solos. Manual de métodos de análise de solo. 2. ed. Rio de Janeiro: EMBRAPA, 1997. 212 p.

FERREIRA, R. R. M.; TAVARES FILHO, J.; FERREIRA, V. M. Efeitos de sistemas de manejo de pastagens nas propriedades físicas do solo. Semina: Ciências Agrárias, Londrina, v. 31, n. 4, p. 913-932, out./dez. 2010.

GREGORICH, E. G.; KACHANOSKI, R. G.; VORONEY, R. P. Carbon mineralization in soil size fractions after various amounts of aggregate disruption. Journal of Soil Science, Oxford, v. 40, n. 3, p. 649-659, sept. 1989.

HILLEL, D. Introduction to soil physics. San Diego: Academic Press, 1982. 364 p.

KASPER, M.; BUCHAN, G. D.; MENTLER, A.; BLUM, W. E. H. Influence of soil tillage systems on aggregate stability and the distribution of $\mathrm{C}$ and $\mathrm{N}$ in different aggregate fractions. Soil \& Tillage Research, Amsterdam, v. 105, n. 2, p. 192-199, sept. 2009.

KEMPER, W. D.; CHEPIL, W. S. Size distribution of aggregates. In: BLACK, C. A. (Ed.). Methods of soil analysis. Madison: American Society of Agronomy, 1965. p. 499-509.

LIMA, J. M.; ANDERSON, S. J.; CURI, N. Phosphateinduced clay dispersion as related to aggregate size and composition in Hapludoxs. Soil Science Society of America Journal, Madison, v. 64, n. 3, p. 892-897, may. 2000.

PINHEIRO, E. F. M.; PEREIRA, M. G.; ANJOS, L. H. C. Aggregate distribution and soil organic matter under different tillage systems for vegetable crops in a Red Latosol from Brazil. Soil \& Tillage Research, Amsterdam, v. 77, n. 1, p. 79-84, may 2004.
RANNO, S. K.; SILVA, L. S.; GATIBONI, L. C.; RHODEN, A. C. Capacidade de adsorção de fósforo em solos de várzea do estado do Rio Grande do Sul. Revista Brasileira de Ciência do Solo, Viçosa, v. 31, n. 1, p. 21 28, fev. 2007.

SÁ, M. A. C.; LIMA, J. M.; SILVA, M. L. N.; DIAS JUNIOR, M. S. Índice de desagregação do solo baseado na energia ultra-sônica. Revista Brasileira de Ciência do Solo, Campinas, v. 23, n. 3, p. 525-531, jul./set. 1999.

SÁ, M. A. C.; LIMA, J. M.; MELLO, C. R. Nível de energia ultra-sônica para estudo da estabilidade de agregados de um Latossolo sob diferentes usos. Pesquisa Agropecuária Brasileira, Brasília, v. 37, n. 11, p. 16491655, nov. 2002.

SANTOS, D.; CURI, N.; FERREIRA, M. M.; EVANGELISTA, A. R.; CANZ FILHO, A. B.; TEIXEIRA, W.G. Perdas de solo e produtividade de pastagens nativas melhoradas sob diferentes práticas de manejo. Pesquisa Agropecuária Brasileira, Brasília, v. 33, n. 2, p. 183-189, fev. 1998.

SANTOS, D. R.; GATIBONI, L. C.; KAMINSKI, J. Fatores que afetam a disponibilidade do fósforo e o manejo da adubação fosfatada em solos sob sistema plantio direto. Ciência Rural, Santa Maria, v. 38, n. 2, p. 576-586, mar./abr. 2008.

SILVA, M. L. N.; CURI, N.; MARQUES, J. J. G. S. M.; GUILHERME, L. R. G.; LIMA, J. M. Ponto de efeito salino nulo e suas relações com propriedades mineralógicas e químicas de Latossolos brasileiros. Pesquisa Agropecuária Brasileira, Brasília, v. 31, n. 9, p. 663-671, set. 1996.

TISDALL, J. M.; OADES, J. M. Organic matter and water-stable aggregates in soils. Journal of Soil Science, Oxford, v. 33, n. 1, p. 141-163, mar. 1982.

WANN, S. S.; UEHARA, G. Surface charge manipulation of constant surface potential soil colloids, I: Relation to sorbed phosphorus. Soil Science Society of America Journal, Madison, v. 42, n. 4, p. 565-570, 1978.

ZELASNY, L. W.; HE, L.; VANWORMHOUDT, A. Charge analysis of soils and anion exchange. In: BIGHAM, J. M. (Ed.). Methods of soil analysis: Part 3 Chemical Methods. Madison, Soil Science Society of America, 1996. p. 1231-1253. 